\title{
Los principios éticos universales y su aplicación a los ensayos clínicos de medicamentos
}

\author{
Universal ethical principles and their application in \\ clinical drug trials
}

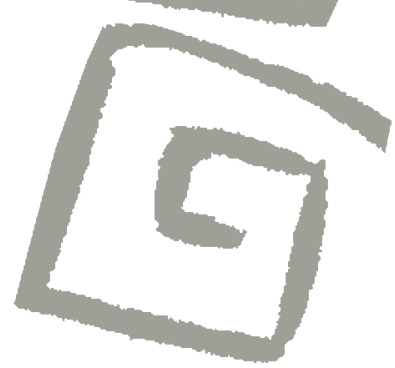

Gonorazky, Sergio Eduardo ${ }^{1}$

'Médico Neurólogo. Especialista Consultor Hospital Privado de Comunidad, Mar del Plata, Buenos Aires, Argentina. egonora@gmail.com
RESUMEN Desde el año 1931 y, especialmente, desde el Código de Núremberg de 1947, un creciente número de declaraciones, regulaciones, normas, guías, leyes, resoluciones y disposiciones pretenden generar condiciones para una mejor protección de los sujetos que participan en estudios de investigación, aunque también algunas implican retrocesos en el respeto a los derechos de poblaciones vulnerables. Sin embargo, todavía no se ha podido evitar la violación de la dignidad de los sujetos de experimentación en ensayos clínicos. Lo que se investiga, cómo se investiga, la calidad y transparencia de los datos obtenidos, el análisis y la publicación de los resultados (tanto de los datos crudos como de los ya elaborados) están sometidos a la lógica del lucro, la cual presenta una tensión permanente con los principios bioéticos y las necesidades de la sociedad. Es necesario el protagonismo activo de los pueblos para que la investigación farmacológica, sus resultados y aplicaciones avancen en un rumbo que subordine el beneficio económico a la protección de los derechos humanos.

PALABRAS CLAVES Investigación; Derechos Humanos; Mala Conducta Científica; Bioética; Comités de Ética en Investigación.

\begin{abstract}
Since 1931, and especially since the Nuremberg Code of 1947, an increasing number of declarations, regulations, norms, guidelines, laws, resolutions, and rules intended to create conditions for better protection of subjects participating in research studies have been published, although some have meant setbacks in the human rights of vulnerable populations. As such, violations of the dignity of experimental subjects in clinical trials continue. What researchers investigate and how the research is done, the quality and transparency of the data, and the analysis and the publication of results (of both raw and processed data) respond to the financial interests of the pharmaceutical companies, coming into permanent tension with bioethical principles and the needs of society. The active participation of civil society is necessary to make it so that pharmaceutical research, results and applications subordinate economic benefits to the protection of human rights.
\end{abstract}

KEY WORDS Research; Human Rights; Scientific Misconduct; Bioethics; Ethics Committes, Research. 


\section{INTRODUCCIÓN}

La investigación en seres humanos tiene una historia tortuosa, con claroscuros permanentes, en la que se destaca, al lado del progreso en el conocimiento, la explotación de individuos vulnerables, ya sea por su condición económica, social o cultural.

Para evitar los abusos, diversos grupos han desarrollado leyes, reglamentos y procesos encaminados a proteger los derechos de los participantes en la investigación. Sin embargo, con frecuencia la investigación clínica se encuentra supeditada a los fines comerciales de las empresas farmacéuticas, las contratadas para investigar -contract research organization (CRO)-, universidades y hospitales que reciben beneficios económicos o equipos, y los investigadores que, además de conseguir prestigio, reciben una elevada remuneración de los patrocinadores de los ensayos.

En este contexto, los sujetos de investigación clínica devienen meros medios, a los que se menoscaba su dignidad con la potencial violación de sus derechos humanos.

El objetivo de este artículo es demostrar que los principios y normas que se han establecido a lo largo de la historia no han conseguido salvaguardar, en la medida esperada, la dignidad y los derechos humanos de los sujetos de investigación clínica, y que la capacidad de reducir los riesgos a los que se exponen ha sido limitada.

La primera sección del artículo presenta un breve recorrido histórico con el objeto de recordar que lo ocurrido durante la investigación en humanos, en la época del nazismo, no sucedió por falta de códigos o regulaciones para controlar posibles abusos de la investigación médica, o porque en la medicina alemana subyacieran elementos particularmente corruptos y monstruosos. Por eso no se puede pensar que, una vez superada la tragedia nazi, el Código de Núremberg y las múltiples declaraciones, regulaciones y normativas hayan logrado controlar los abusos.

En la segunda sección del artículo se discuten los intereses económicos que intervienen en el desarrollo de terapias medicamentosas y que pueden llevar a los patrocinadores de los ensayos clínicos y a los investigadores a violar los derechos humanos de los sujetos y la metodología de los estudios. El interés económico puede sobrepasar el interés de encontrar soluciones a los problemas de salud que más afectan a la población y promover el sesgo en las publicaciones científicas.

Finalmente, se examinan los organismos de control de la investigación y de la comercialización de fármacos de los países productores de medicamentos innovadores -específicamente, la Food and Drug Administration (FDA) de EE.UU. y la European Medicines Agency (EMA)- para determinar si son lo suficientemente confiables como para que los países receptores de medicamentos puedan certificar automáticamente cualquier producto aprobado por ellos.

\section{HISTORIA DE LA INVESTIGACIÓN CLÍNICA Y EL INICIO DE LAS NORMATIVAS}

La tensión entre el interés por el avance de la ciencia y la necesidad de proteger a los sujetos de investigación no es algo nuevo y ha penetrado las diferentes especialidades de la investigación clínica en todos los continentes.

\section{Los estudios sobre la sífilis y la respuesta del gobierno prusiano}

En 1898, Albert Neisser, quien en el año 1879 descubrió el agente transmisor de la gonorrea (llamado en su honor Neisseria gonorrhoeae), publicó un ensayo clínico en el que, buscando un método para prevenir la sífilis, inyectó suero libre de células de pacientes con esta enfermedad en personas hospitalizadas por otros problemas de salud. La mayoría de estos enfermos eran prostitutas y a ninguna se le solicitó consentimiento para participar de dicho experimento. Algunas contrajeron la enfermedad, por lo que Neisser concluyó que el experimento había fracasado y que la sífilis la habían contraído por su condición de prostitutas (1).

El debate público que generó este caso motivó que la fiscalía lo investigara y que la Corte Real de Disciplina de Prusia sancionara al investigador por no haber obtenido el debido consentimiento informado, aunque el estudio no hubiese representado ningún riesgo para los participantes (1). 
En 1899, el Parlamento Prusiano discutió el problema y, seguidamente, el Ministerio de Asuntos Religiosos, Educacionales y Médicos encargó un informe a la Oficina Médica Científica de Salud, de la que formaban parte prestigiosos médicos alemanes, entre ellos Rudolf Virchow. La comisión concluyó que una investigación que coloca al sujeto en riesgo de contraer una infección no debe ser efectuada, y que la investigación debe ser siempre precedida por un adecuado consentimiento informado. El ministerio también solicitó consejo legal. Los juristas afirmaron que, según las leyes criminales, realizar una investigación no-terapéutica en un sujeto sin consentimiento cumplía los criterios de injuria física y que la validez científica del experimento no mitigaba el daño. Los problemas de coerción, persuasión y la desigual autoridad entre médicos y pacientes se discutieron en detalle y los juristas concluyeron que los derechos y la moralidad tenían la misma importancia para el género humano que el progreso científico (1).

Como consecuencia de estos planteos, en 1900, el ministerio envió directivas a todos los hospitales y clínicas. Los directores médicos fueron advertidos de que las intervenciones médicas distintas al diagnóstico, tratamiento e inmunización debían excluir a todo "menor o incompetente por otras razones", así como a todo sujeto que no hubiera dado un consentimiento "no ambiguo" tras una "adecuada explicación de las consecuencias negativas de la intervención". Cualquier investigación debía ser realizada con la autorización del director médico, y en todos los casos el cumplimiento de estos requerimientos debía quedar "documentado en la historia clínica" (1).

\section{La fiebre amarilla}

En 1881, Carlos Finlay dio a conocer su teoría sobre el papel del mosquito hembra Aedes aegypti en la transmisión de la fiebre amarilla y las pruebas favorables, aunque no eran totalmente concluyentes (2). En dicho estudio incluyó a cinco individuos (uno de ellos era él mismo) y los expuso a la picadura de un mosquito que previamente había picado a individuos enfermos. De los cinco individuos, tres contrajeron la enfermedad, no así él ni un quinto, aunque todavía no había transcurrido el tiempo necesario para determinar si el último había o no contraído la enfermedad. Ninguno de los que contrajo la enfermedad falleció. Antes de comenzar el relato de sus experimentos expresó que había obtenido las debidas autorizaciones, pero no explicitó en qué consistieron.

En 1897, el bacteriólogo italiano Giuseppe Sanarelli, radicado en Montevideo, postuló que la fiebre amarilla estaba producida por un bacilo que él manifestaba haber descubierto y, para probarlo, inyectó, sin consentimiento, cultivos del supuesto bacilo en cinco individuos internados por otras causas, tres de los cuales murieron. Según Sanarelli, los individuos contrajeron una enfermedad que describió como una "clásica fiebre amarilla". Un año después de publicado el trabajo de Sanarelli, Osler rechazó absolutamente esta investigación expresando que "inyectar deliberadamente un veneno del que se sabe tiene un alto grado de virulencia en un ser humano, a menos que $U d$. haya obtenido la autorización de este hombre, no es ridículo, es criminal" (3).

Walter Reed, Arístides Agramonte, James Carroll y Jesse Lazear confirmaron la teoría de Finlay sometiendo a voluntarios sanos al contacto con fómites de pacientes infectados, a la inyección endovenosa de sangre de infectados o a la inoculación con mosquitos infectados (produciendo la enfermedad en las dos últimas situaciones, pero no en la primera). Los experimentos fueron realizados con consentimiento informado y con una compensación económica. En dicho consentimiento se les advertía que corrían riesgo de muerte. Sin embargo, 15 de los voluntarios eran hombres enlistados y uno un oficial, y hoy serían considerados población vulnerable. Los restantes eran dos civiles de origen americano y 15 inmigrantes españoles. Lazear, uno de los investigadores, falleció de fiebre amarilla, ningún otro de los participantes en el estudio tuvo un desenlace fatal (3).

El consentimiento informado que se entregaba en inglés o español, según el caso, a los voluntarios expresaba: "Quien suscribe entiende perfectamente bien que en caso de desarrollar fiebre amarilla, pone en riesgo en cierta medida su vida; pero que siendo enteramente imposible para él evitar la infección durante su estadía en la isla, prefiere correr el riesgo de contraer ésta intencionalmente con el convencimiento que recibirá de la Comisión el mayor cuidado y los mejores servicios médicos expertos" (3). 


\section{La normativa existente en Alemania y los experimentos nazis}

En 1931, en Alemania, una circular del ministerio del interior del gobierno estableció las "Guías para nuevas terapias en la experimentación con humanos" (4). Más allá de afirmar los principios de beneficencia y no maleficencia, las regulaciones estaban basadas en el principio de autonomía del paciente y en la doctrina legal del consentimiento informado. Además, diferenciaba el experimento con nuevas terapias para el tratamiento, diagnóstico o prevención de enfermedades de la experimentación en humanos con propósitos no terapéuticos, que incluía el estudio de efectos adversos y consecuencias que no podían ser adecuadamente determinadas en el marco de los conocimientos de aquel entonces.

Las Guías establecían que: 1) las nuevas terapias solo podían ser introducidas sin consentimiento para salvar vidas o prevenir daños severos a la salud si su administración era pospuesta, pero la investigación no terapéutica, no podía realizarse sin consentimiento bajo ninguna circunstancia; 2) la ética médica rechazaba cualquier explotación por necesidades sociales o económicas en la conducción de estos experimentos; 3 ) el tratamiento de niños y menores de 18 años requería un especial cuidado; y 4) la utilización de microorganismos vivos exigía mantener una elevada cautela (4).

En 1939, comienza en Alemania el mal Ilamado programa de "eutanasia". En ese marco, se desarrollaron los programas de investigación en humanos y algunos investigadores pretendieron sacar provecho indirecto del exterminio de "vidas indignas de ser vividas" (concepto por medio del cual el nazismo intentó fundamentar "científicamente" la necesidad de apartar de la sociedad a quienes ingresaban en esta categoría, y que comenzó con la esterilización en masa de supuestos portadores de taras genéticas, continuó con el asesinato de niños y luego de adultos discapacitados, para culminar con el asesinato en masa de los considerados racialmente inferiores, opositores políticos, homosexuales, "religiones peligrosas", etc.) (5).

Uno de los ejemplos más notables es el de Julius Hallervorden del hospital estatal de Brandenburgo, un neuropatólogo internacionalmente conocido de quien dependía la institución de cuidados crónicos Brandenburg-Grönen, uno de los seis centros que desarrolló el programa de eutanasia. Esta situación le brindó la posibilidad de estudiar la neuropatología de enfermedades raras en gran escala.

Para mejorar la calidad de sus estudios, Hallervorden examinaba personalmente a los pacientes antes de ser ejecutados para, posteriormente, obtener sus cerebros. Su indiferencia moral ante el destino de los pacientes fue descripta después de la guerra por Leo Alexander, neurólogo norteamericano y refugiado de Europa Central, a quien Hallervorden le comentó:

Yo escuché lo que ellos iban a hacer por eso fui y les dije: "Vean muchachos, si ustedes van a matar a estos individuos, al menos tomen sus cerebros de tal manera que el material sea utilizable". Había un maravilloso material entre esos cerebros, hermosos defectos mentales, malformaciones y enfermedades infantiles de comienzo temprano. Ellos me preguntaron: "¿Cuántos desea usted examinar?" Y yo les contesté que un número ilimitado: cuantos más, mejor. $\mathrm{Y}$ acepté los cerebros, por supuesto. De dónde provenían y cómo provenían no era realmente mi problema. (6)

\section{Los experimentos japoneses en China y Manchuria}

Menos publicitados, pero igualmente atroces, fueron los experimentos médicos realizados por el Imperio Japonés en Manchuria y China, con miles de víctimas. Desde provocar heridas de bala en prisioneros para el entrenamiento de los cirujanos del ejército, pasando por el desarrollo de armas biológicas, estudios de enfermedades infecciosas con introducción de gérmenes y posterior vivisección y muerte de sujetos de investigación, y estudios fisiológicos de exposición a bajas presiones o bajas temperaturas en los que se incluían bebés. Muchos de estos investigadores publicaron sus trabajos años después de la guerra. Uno de ellos, Hisato Yoshimura, llegó a ser presidente de la Kyoto Prefectural University of Medicine y laureado por el emperador Hiroito en 1978 con la Orden de Tercera Clase del Sol Naciente, por sus trabajos pioneros en la "ciencia de adaptación 
ambiental" $(7,8)$. La apatía moral y la impunidad se visualiza claramente al contrastar la recepción del premio con las declaraciones en el Juicio de Khabarovsk (ex Unión Soviética), en 1949, del sargento mayor de la Policía Militar, Satoru Kurakazu:

Cuando ingresé en el laboratorio de la prisión, cinco individuos de origen chino, sujetos de experimentos, estaban sentados en un largo banco; dos de estos chinos no tenían dedos en absoluto, sus manos estaban negras; en los otros tres, los huesos eran visibles, tenían sus dedos, pero ellos eran solo huesos. Yoshimura me dijo que esto era consecuencia de los experimentos con frío. (8)

\section{EL DESARROLLO DE LA NORMATIVA INTERNACIONAL}

A partir del fin de la Segunda Guerra Mundial se han formulado códigos, declaraciones, normativas, leyes y reglamentaciones tendientes a proteger los derechos de los pacientes/participantes en los estudios, con el objetivo de evitar que se repitieran las atrocidades ocurridas. Muchas han significado avances en la protección de la integridad de las personas; otras, retrocesos producto de la presión ejercida por las necesidades lucrativas de las farmacéuticas innovadoras, aunque disimuladas bajo el disfraz de los costos del desarrollo científico.

Forman parte de la normativa: 1) el Código de Núremberg; 2) la Declaración Universal de Derechos Humanos, de la Organización de las Naciones Unidas (ONU); 3) la Declaración de Helsinki, de la Asociación Médica Mundial (AMM) con sus sucesivas modificaciones; 4) el Informe Belmont; 5) las Guías Éticas Internacionales, del Council for International Organizations of Medical Sciences (CIOMS); 6) la Declaración Universal sobre Bioética y Derechos Humanos, de la UNESCO; 7) la Declaración Universal sobre el Genoma Humano y los Derechos Humano, de la UNESCO; 8) la Convención sobre Derechos Humanos y Biomedicina, del Concejo Europeo; y 9) la Guía de Buenas Prácticas Clínicas, de la Organización Panamericana de la Salud (OPS).
En América Latina ha habido una copiosa producción de leyes, regulaciones y normas sobre investigación médica en humanos específicamente orientada a evitar que se violen normas éticas y los derechos humanos de los sujetos de experimentación. En casi todos los países de la región ha habido avances seguidos de retrocesos dependientes de las presiones que ejercen los patrocinadores de los ensayos clínicos y sus investigadores. Una obra reciente presenta los avatares de la legislación en Argentina, Brasil, Costa Rica, México y Perú, países en donde se desarrollan cerca del $80 \%$ de los ensayos clínicos de la región (9).

\section{A pesar de las normas, persisten las violaciones}

Los investigadores suelen encontrar justificaciones para violar la dignidad de las personas al hacer consideraciones científicas. En 1967, Thomas Rivers, el afamado virólogo que dirigió el Instituto Rockefeller para la Investigación Médica de Nueva York, escribió en sus memorias:

Todo lo que yo puedo decir es que está contra la ley hacer muchas cosas, pero la ley cierra los ojos cuando un hombre reputado quiere hacer un experimento científico. Por ejemplo, el código criminal de la Ciudad de Nueva York sostiene que es un crimen inyectar a una persona material infeccioso. Bien, yo probé una vacuna para la fiebre amarilla con material vivo en mi pabellón del Hospital Rockefeller. No es un secreto, y puedo asegurarle que las personas en el Departamento de Salud de la Ciudad de Nueva York sabían lo que se hacía [...] A menos que la ley cierre los ojos ocasionalmente, usted no tendría progreso en la medicina. (10)

A continuación, por razones de espacio, presentamos de forma resumida unas pocas violaciones, algunas ampliamente documentadas.

\section{Investigaciones en EE.UU.}

El caso del estudio observacional de sífilis en Tuskegee, Alabama, aunque comenzó antes de los experimentos nazis, se menciona en los 
textos como ejemplo paradigmático de violaciones pos-Núremberg, ya que se prolongó desde 1932 hasta 1972. Los investigadores pretendían evaluar la evolución natural de la sífilis en 400 hombres negros. Fue diseñado por el Servicio de Salud Pública de EE.UU., y en los últimos años del estudio se negó el acceso de los sujetos a un tratamiento eficaz (11-13).

Esa misma agencia también participó en estudios en Guatemala que involucraron la inoculación de treponema pallidum, agente infeccioso de la sífilis, y otros gérmenes causantes de infecciones de transmisión sexual, así como en experimentos con tratamientos sin obtener previamente el consentimiento informado y sin ofrecer un adecuado seguimiento posterior (1946-1948). Un total de 5.500 guatemaltecos fueron sujetos de la investigación, incluyendo prisioneros, prostitutas, enfermos mentales y soldados (10), de los cuales 1.300 fueron infectados deliberadamente con sífilis, gonorrea y otras enfermedades de transmisión sexual. Unos 700 recibieron algún tratamiento. Aproximadamente 83 habían fallecido hacia fines de 1953 (14).

En los controvertidos estudios realizados en el asilo de Willowbrook se infectó intencionadamente a niños con discapacidad mental con el virus de la hepatitis (1963-1966). Los estudios permitieron diferenciar dos formas de hepatitis (hoy Ilamadas A y B) (15). Sin embargo, los aspectos éticos denunciados por Beecher, en su célebre escrito sobre ética e investigación clínica (16), siguen vigentes, más allá de las discusiones inacabadas sobre el tema (17-19). La realidad es que la investigación de Willowbrook solo puede justificarse éticamente desde una estrecha perspectiva consecuencialista y minimalista, dado que no pretendió hacer nada para resolver, por ejemplo, los problemas de hacinamiento que existían en dicha institución.

En 1963, en el Hospital Judío de Enfermedades Crónicas de Nueva York se inyectaron células cancerosas en 22 pacientes debilitados, con el objeto de investigar la relación entre la inmunodepresión y el cáncer (20). La investigación, que claramente quebrantaba la regla de "primero no hacer daño" por el riesgo que ella misma entrañaba, se efectuó sin el consentimiento informado de los sujetos de investigación.

Desde mediados de 1950 hasta mediados de 1980, el ejército norteamericano, buscando armas químicas más humanas que las balas y metralletas, experimentó en secreto en soldados (21). Se pretendía luchar contra el enemigo con nubes de químicos psicotrópicos que incapacitan la mente durante un periodo de tiempo. Los experimentos se condujeron en un centro de investigaciones, $y$ en miles de soldados insuficientemente informados se probaron substancias químicas entre ellas gases lacrimógenos, LSD, bencilato de 3-quinuclidinilo (BZ) y VX, otro agente altamente letal para el sistema nervioso que fue desarrollado en el centro de investigaciones. La especialidad del director militar de las investigaciones era una familia de moléculas que bloqueaban un neurotransmisor importante, y causaban delirio. Para los experimentos con sarín se contrataron investigadores de la Universidad de Johns Hopkins. Las drogas se conocían básicamente por los códigos del ejército y eran secretas. A los soldados nunca se les decía lo que les daban o cuáles podrían ser los efectos específicos, y el ejército no se molestó en hacer un seguimiento de los sujetos que habían participado en los experimentos.

\section{Ensayos clínicos en África y América Latina}

Las violaciones en África han sido tan frecuentes que dieron material para la famosa novela de John Le Carré, El jardinero fiel. En un ensayo clínico, Pfizer debió llegar a un acuerdo de indemnización y reconocimiento por la realización sin consentimiento de una investigación con trovafloxacina (Trovan) en niños durante una epidemia de meningitis en Nigeria en el año 1996, aunque por un valor notoriamente inferior al originalmente reclamado $(22,23)$. La historia de este ensayo tiene todos los ingredientes de violación de los derechos humanos: 1) ausencia de adecuado consentimiento informado (Pfizer adujo como argumento que no existía una norma internacional que exigiera establecer el consentimiento informado en ensayos experimentales de fármacos en África) (24); 2) administración de una dosis inferior a la recomendada del comparador (ceftriaxona); 3) un seguimiento incompleto durante el tratamiento e inexistente con posterioridad a este (25-27); el patrocinador recurrió a investigadores para averiguar vínculos de corrupción del fiscal general para ponerlo en evidencia y que no lleve adelante las querellas (28); y 4) pérdida de los registros clínicos 
de los niños que participaron en el estudio (23); 11 habrían muerto y otros habrían quedado con secuelas neurológicas (27).

En Argentina se han registrado violaciones éticas y normativas que tomaron estado público y requirieron la intervención de la justicia. Entre ellas se pueden mencionar estudios oncológicos, realizados sin aprobación de la Administración Nacional de Medicamentos Alimentos y Tecnología (ANMAT), y ensayos clínicos que violaron otras regulaciones. Entre estos últimos se puede mencionar el estudio Clinical Otitis Media \& Pneumonia Study (COMPAS) de vacuna contra el neumococo, realizado en las provincias de Córdoba, Santiago del Estero (la más pobre del país), Mendoza y San Juan, con 13.981 niños reclutados (29) que terminó en el Poder Judicial de la Nación con multas a la empresa y a los investigadores principales.

El Comité Hospitalario de Ética y el Consejo de Revisión de Estudios de Investigación del Hospital Privado de Comunidad de Mar del Plata (Argentina) rechazaron un estudio en el que se pretendía investigar la eficacia y seguridad de una quinolona (la moxifloxacina, un medicamento del mismo grupo que la trovafloxacina) en sujetos pediátricos con infección intraabdominal complicada, el cual adolecía de severas fallas éticas y metodológicas (30) y que exponía a riesgos desproporcionados a pacientes vulnerables en una patología para la que hay tratamiento eficaz demostrado. Este ensayo se ha implementado en varios países, incluyendo EE.UU.

Otros muchos ejemplos de violaciones éticas y normativas en ensayos clínicos en Argentina, en Brasil, Costa Rica, México y Perú se han descrito en el libro Ética y Ensayos Clínicos en América Latina (31).

\section{Investigaciones con doble estándar}

En 1994 se publicó el estudio AIDS Clinical Trials Group (ACTG) protocolo 076 que determinó que la administración preparto, intraparto y al neonato de zidovudina reduce el riesgo de transmisión materno-fetal del VIH en aproximadamente un $67 \%$ (32). En el año 1997, Lurie y Wolfe (33) identificaron 18 estudios controlados, desarrollados luego de la publicación del estudio ACTG protocolo 076 , que involucraron a 17.000 mujeres. En los dos estudios realizados en EE.UU., el acceso a la zidovudina fue irrestricto. En 15 de los 16 estudios realizados en países en desarrollo, no todos los pacientes tuvieron acceso a la zidovudina (sea porque se investigó la zidovudina en regímenes menos costosos y complejos contra placebo o porque se investigaron otros métodos de prevención para la transmisión del HIV). Dos de estos trabajos, zidovudina contra placebo, fueron publicados en una revista de prestigio como The Lancet $(34,35)$.

No quedan dudas del carácter antiético de estos 15 estudios que, como bien señalan Lurie y Wolfe (33), transgredieron las normas de las Pautas Éticas Internacionales para la Investigación Biomédica en Seres Humanos del CIOMS de 1993 que expresa que:

\footnotetext{
Un organismo patrocinador externo debe someter el protocolo de investigación para su evaluación ética y científica según las normas del país del organismo patrocinador, y las normas éticas aplicadas no deben ser menos exigentes de lo que serían en el caso de que la investigación se llevara a cabo en ese país. (36)
}

O, como manifiesta Marcia Angell (37), violan los principios de la Declaración de Helsinki de 1989, uno de los cuales expresa que "En cualquier estudio médico, debe asegurarse a todos los pacientes, incluyendo los del grupo control, si lo hay el mejor método diagnóstico o terapéutico probado" (38).

Este doble estándar en investigación fue justificado por los investigadores y por las agencias reguladoras con el argumento de que la norma de los países en los que se hacía la investigación era la "ausencia de tratamiento". Este razonamiento explota la situación de inequidad en el acceso a los tratamientos para desarrollar estudios que terminan siempre favoreciendo a la industria y no resuelven nunca los problemas de injusticias y asimetrías en la distribución de los resultados de las investigaciones.

El problema de doble estándar en investigación, así como el del acceso a los mejores tratamientos y métodos descubiertos luego del ensayo clínico, motivó un profundo debate en el ámbito bioético. Sucesivas modificaciones de la Declaración de Helsinki (la última del año 2013) fueron objeto de controversias que están más allá 
del objetivo del presente escrito. Sin embargo, no debe dejar de registrarse que los dos problemas mencionados (doble estándar y acceso posinvestigación), nos indican la clara intención de los países de altos ingresos de naturalizar la explotación de la población de los países de bajos y medianos ingresos y, eventualmente, también la de la población de sus propios países que no tienen acceso a los mejores tratamientos probados.

En 2001, la International Conference on Harmonisation of Technical Requirements for Registration of Pharmaceuticals for Human Use $(\mathrm{ICH})$, que está conformada por las agencias reguladoras de EE.UU., de los países de la Unión Europea en donde se encuentra la gran industria innovadora y Japón, así como representantes de la industria farmacéutica de dichos países, publicó la guía Choice of Control Group in Clinical Trials (39) y en el punto 2.1.3 expresa que:

\footnotetext{
Cuando se prueba un nuevo tratamiento para una condición en la cual no hay un tratamiento efectivo conocido, no existe usualmente un problema ético en comparar el nuevo tratamiento al placebo. El uso de un placebo como control puede generar problemas éticos de aceptabilidad y factibilidad cuando un tratamiento efectivo está disponible para la condición bajo estudio en el ensayo propuesto. En los casos en que hay un tratamiento disponible y conocido para prevenir un daño serio, tal como muerte o morbilidad irreversible en la población bajo estudio, es generalmente inapropiado usar el placebo como control. (39 p.16)
}

A esto se agrega en el 2.1.7.1:

Cuando se conoce y existe una terapia efectiva para prevenir la muerte o una morbilidad irreversible para una población particular, esta población no puede ser estudiada en un ensayo clínico controlado contra placebo; la condición particular y la población para la cual esto es verdadero puede ser objeto de controversia. (39 p.20)

Es decir, deja abierta la posibilidad de utilizar terapias probadas que son efectivas en poblaciones particulares en las que no exista la terapia efectiva para prevenir la muerte o morbilidad irreversible.

\section{El ensayo clínico con surfactante en América Latina}

De características similares fue la controversia generada por un protocolo de investigación de surfactantes vs. placebo que estaba siendo considerado por la FDA para su aprobación en el año 2001, para ser implementado en cuatro países de Latinoamérica (40), en el que se iban a inscribir a centenares de bebés prematuros, siendo que existían tratamientos probados que reducían la mortalidad relativa en un $37 \%$, la hemorragia intraventricular en el período neonatal en un $12 \%$ y la mortalidad en el primer año de vida en un $20 \%$ (41). Llamativamente la compañía que iba a realizar el estudio contra placebo en América Latina, planificaba, al mismo tiempo, un estudio contra un surfactante aprobado por la FDA en Europa. Ante la denuncia de Public Citizen y de ONG latinoamericanas, finalmente el estudio en América Latina se llevó adelante en condiciones similares a las europeas (42).

No casualmente la FDA, en el año 2008, decidió no exigir más la adhesión a la Declaración de Helsinki en estudios realizados fuera de EE.UU. y solo exigir el cumplimiento de las guías de la $\mathrm{ICH}(43,44)$.

Los hechos relatados no son anécdotas aisladas. Numerosos casos son denunciados en publicaciones y libros dedicados a estos temas $(45,46)$.

\section{LOS INTERESES DE LA INDUSTRIA FARMACÉUTICA INNOVADORA}

\section{La investigación y las necesidades de la población}

Mientras abundan los tratamientos para ciertas enfermedades, hay otras, las Ilamadas olvidadas tales como la malaria, filariasis, oncocercosis, tracoma, tripanosomiasis, leishmaniosis, dengue, mal de Chagas, etc., que causan 35.000 muertes por día en países de bajos y medianos ingresos, con morbilidad relevante y que siguen sin tratamiento (47).

La falta de inversión en tratamientos para enfermedades que afectan a poblaciones sin capacidad de compra es conocida. Entre los años 1975 y 1999, se introdujeron al mercado 1.393 nuevas 
entidades químicas o medicamentos (NEQ), de los cuales solo 16 estaban indicados para estas enfermedades olvidadas (48). En el año 2004, los gastos en investigación sobrepasaron los 100.000 millones de dólares, pero solo el $10 \%$ de esa suma se destinó a la investigación de enfermedades responsables del $90 \%$ de la carga de enfermedad (49). Esta disparidad ha sido Ilamada la brecha 90/10 y superarla es uno de los grandes desafíos de la humanidad. La contradicción existente entre la lógica del lucro que incentiva la investigación y la de las necesidades de quienes requieren nuevos tratamientos, pero carecen de recursos para pagarlos, explica la paradoja.

Entre 1998 y 2003, la FDA aprobó 487 drogas. De ellas, 379 (78\%) fueron consideradas por la agencia como poseedoras de cualidades terapéuticas similares a una o varias que estaban en el mercado (drogas me-too) y 333 (68\%) eran nuevas formulaciones de viejas combinaciones. Solo 67 (14\%) de las 487 eran medicamentos auténticamente innovadores (50).

La trágica epidemia ocasionada por el virus del Ebola en 2014, con miles de muertos y una letalidad aproximada del 55\%, nos trae nuevamente a la realidad del descalabro éticamente injustificable del actual sistema de investigación y desarrollo de medicamentos. Según Donovan: "La sombría realidad es que las compañías farmacéuticas están en el negocio de producir tratamientos que la gente pueda pagar" (51) y, si bien este autor pretende justificar el hecho desde el alto costo de la investigación, la realidad es que las ganancias de la industria farmacéutica superan ampliamente los costos de la investigación (52). Sin embargo, Donovan tiene razón cuando afirma que si la enfermedad se traslada a los países desarrollados: "Podría demandarse una intervención más efectiva, lo que nos lleva a la odiosa sospecha de que el mundo se preocupa menos cuando el problema está confinado a los pobres países africanos..." (51).

\section{La falta de transparencia en la información de resultados}

En 2009 se produjo el brote epidémico por el virus de la influenza A (H1N1). La OMS, los Centers for Disease Control and Prevention (CDC) y la American Academy of Pediatrics de EE.UU., el National Institute for Health and Care Excellence (NICE) de Gran Bretaña, entre otros, recomendaron la utilización de inhibidores de la neuraminidasa para la prevención de complicaciones en pacientes con alto riesgo de contraer la enfermedad (53). Estas recomendaciones se hicieron a pesar de no tener evidencia suficiente sobre sus beneficios $y$ riesgos, y determinaron que los gobiernos gastasen miles de millones de dólares para tener suficiente stock de estos medicamentos ante la anunciada pandemia (54).

Cuando la totalidad de los datos estuvieron al alcance de los especialistas se pudo concluir, por un lado, que no había evidencia de que la administración de estos fármacos redujera el tiempo de hospitalización o las complicaciones y, por otro, que sí incrementaba los efectos adversos $(55,56)$. La cuestionable calidad de los datos en los estudios realizados (54), así como el sesgo en las conclusiones de los ensayos publicados y la información no compartida (57) permiten afirmar que se dilapidaron 20.000 millones de dólares (58), que resultaron en abundantes ganancias para la industria. Un escándalo poco conocido por el público.

El caso de rofecoxib (Vioxx) ha sido ampliamente documentado (59). La manipulación de los datos de uno de los ensayos clínicos, la falta de transparencia de la empresa y la complicidad de la FDA se descubrieron cuando un juez solicitó información en el transcurso de juicios que pacientes o sus familias iniciaron cuando ya no se pudieron encubrir los efectos secundarios y la mortalidad que produjo el medicamento. Desde que rofecoxib salió al mercado hasta que se retiró en 2004, Merck facturó un promedio de 2.500 millones de dólares anuales. Se estima que 100 millones de pacientes recibieron el medicamento. Se calcula que entre 88.000 y 139.000 personas padecieron un infarto agudo de miocardio a consecuencia del rofecoxib y que, de ellas, entre el $30 \%$ y el $40 \%$ fallecieron por esta causa $(60,61)$.

Otro ejemplo de sesgo intencional es lo ocurrido con el uso de la paroxetina para tratar la depresión en población pediátrica. Un estudio que se llevó a cabo en EE.UU. entre los años 1993 y 1996 no mostró que fuese superior al placebo (estudio 329) y otro que se implementó en Europa, Sudamérica y otros lugares mostró que era inferior al placebo (estudio 377) (62). Sin embargo, 
un equipo del laboratorio SmithKline Beecham recomendó publicar datos positivos pues "sería inaceptable incluir una afirmación de que la eficacia no había sido demostrada, puesto que esto podría socavar la confianza en la paroxetina" por lo que se envió un resumen favorable sobre el fármaco a la reunión del European College of Neuropsychopharmacology en 1998 (62). A pesar de que la publicación informó que la paroxetina generalmente es bien tolerada y efectiva para la depresión mayor en adolescentes, posteriormente se desaconsejó su uso en menores de 18 años por incrementar el riesgo de suicidio entre un $50 \%$ y un $200 \%$ en comparación con el placebo (62).

La manipulación de los datos puede observarse tanto en las conclusiones observadas con relación a inhibidores de recaptación de la serotonina y el riesgo de conductas suicidas, así como en el fracaso de las entidades regulatorias en detectar el problema $(63,64)$.

En las últimas dos décadas, expresa Angell (65), los avances de la industria farmacéutica en la evaluación de sus propios productos no tienen precedentes y los sesgos observados en los ensayos con rofexocib "no son inusuales ni tampoco limitados a Merck". En los estudios patrocinados por la industria, esta "es la dueña de la base de datos y tiene la discreción de determinar quién tendrá acceso [...] En este punto, los investigadores tienen las manos atadas, proveen de pacientes y coleccionan datos de acuerdo al protocolo de la compañía" (65). Los investigadores son más bien técnicos encargados de la recolección de datos, pues no participan ni del diseño ni del análisis de la investigación (66).

Hay muchas maneras de producir sesgos en los resultados sin falsificar los datos del documento fuente o de las bases de datos, tales como decidir realizar el análisis "bajo tratamiento" o por "intención de tratamiento" (67), comparar con placebo (aun cuando exista una droga con eficacia comprobada), o utilizar una dosis más baja del comparador, o una dosis más alta para incrementar los efectos adversos de este y minimizar los de la droga investigada, u ocultar parte de los datos, entre otros (65).

Diversos autores señalan las limitaciones en el acceso a los datos y el sesgo en la publicación de los resultados (68). Sin embargo, es notable que las propias agencias reguladoras sean parte del ocultamiento de la información. Goldacre relata las dificultades que tuvieron investigadores del Centro Cochrane Nórdico para conseguir los datos relacionados con los medicamentos para adelgazar -orlistat y rimonabant- y los tres años y medio que les llevó alcanzar el objetivo. Cabe destacar que durante la espera hubo que retirar uno de los medicamentos del mercado por sus efectos adversos (24). Resulta auspiciosa la movilización creciente para conseguir que la industria haga accesible a investigadores independientes los datos crudos de los pacientes, manteniendo su privacidad, y no simplemente los resultados ya analizados (69-72)

Angell califica a la investigación, producción, promoción y venta de fármacos como un sistema resquebrajado que alcanza a las agencias reguladoras (65). Gotzsche, miembro del Centro Cochrane Nórdico, como resultado de una investigación en la que relata fraudes, ocultamiento de información sobre daños, tergiversación de los resultados de las investigaciones, promoción de medicamentos con resultados de eficacia no probadas y fuera del prospecto, afirmó que el crimen corporativo en la industria farmacéutica es frecuente, importante y repetitivo (53).

\section{La capacidad de las agencias para proteger a los sujetos de investigación y la calidad de la información}

Angell comenta que la FDA padece las presiones de la industria a través de sus dieciocho comités de asesores permanentes que evaluan el registro de nuevos medicamentos, ya que muchos miembros de esos comités tienen vínculos financieros con las compañías interesadas. Citando a USA Today, comenta que "en el $92 \%$ de las reuniones, al menos un miembro tenía un conflicto de interés financiero" y que "en el 55\% de las reuniones, la mitad o más de los asesores de la FDA tenían conflictos de intereses" $(52,73)$.

La colusión de intereses alcanza también a la EMA. Goldacre relata que, en diciembre de 2010, Thomas Lönngren abandonó su cargo de director ejecutivo de la EMA y envió una carta al consejo de administración anunciando que se disponía a aceptar una posición de asesor en la industria farmacéutica en un plazo de cuatro días (24). 
David Healy manifiesta que comúnmente se asume que la FDA almacena los datos crudos de los ensayos, de tal manera que si alguien observa un problema en un tratamiento, puede consultar allí lo que le preocupa, pero esto no es así (64). Agrega que si bien las compañías envían a la FDA el archivo de datos, esta no analiza los datos en bruto sino que se limita a trabajar sobre las tablas que las empresas confeccionaron (64). Además menciona que la FDA funciona como un auditor: realiza muestreos de los registros clínicos para determinar en qué medida se corresponden con las figuras y las tablas que la industria confeccionó y si requiere más análisis le pide a la compañía que lo efectúe.

La ineficacia de las agencias reguladoras podría parecer una exageración. Sin embargo, una auditoría del inspector general del Department of Health and Human Services (HHS) de EE.UU. que cubrió el periodo de 2000 a 2005 concluyó que los funcionarios de la FDA desconocían cómo se estaban haciendo muchos ensayos clínicos, pues auditaban menos del $1 \%$ de los sitios en los que se realizaban los ensayos (74); y que la FDA no solo monitoreó pocos Comités de Ética en Investigación (CEI), sino que descuidó considerablemente la protección de los sujetos de investigación.

La auditoría concluyó que la FDA: 1) carecía de un registro de los ensayos clínicos en proceso y de CEI; 2) carecía de una base de datos en la que se registrasen las inspecciones de las investigaciones biomédicas, lo que le impedía rastrearlas adecuadamente; 3 ) confiaba en el acatamiento voluntario de las medidas correctivas por violación relevante de la regulación; y 4) mantenía ambigüedad y ausencia de coordinación, lo que impedía llevar adecuadamente las inspecciones de investigaciones biomédicas (74).

Una segunda auditoría del HHS a la FDA, en 2008, cuestionó la calidad de los datos, de investigaciones realizadas en el extranjero, que remiten los patrocinadores para obtener la comercialización de drogas en EE.UU., y la profundidad con que la FDA monitorea e inspecciona esas investigaciones (75). La auditoría concluyó que el 80\% de las drogas y productos biológicos aprobados descansan en datos brindados por los patrocinadores y provienen de ensayos clínicos realizados fuera de EE.UU.; además dijeron que, en ese año, la FDA inspeccionó menos del $1 \%$ de los sitios en que se llevaban adelante dichos ensayos (75). Cuando la FDA y la EMA aprueban los medicamentos, las agencias reguladoras de otros países como Argentina o México también los aprueban, creyendo que estas agencias son eficaces en su accionar.

Los CEI, por su parte, pueden constituir excelentes instrumentos destinados a la protección de los pacientes/participantes en estudios de investigación. Sin embargo, también pueden servir para legitimar estudios en los que se transgreden normas, resoluciones, disposiciones, leyes $y$ principios generales destinados a salvaguardar la dignidad de los pacientes (76), ya sea en los protocolos, las hojas de información, el consentimiento informado o en el desarrollo de la investigación. Una investigación clandestina de la Government Accountability Office (GAO) de EE.UU., en la que se presentó un protocolo increíblemente absurdo a tres institutional review board (comités de revisión institucional), documentó que uno de los comités aprobó el estudio en menos de una semana y los otros dos lo rechazaron, lo que revela los claroscuros de las revisiones éticas (77). El comité, con fines de lucro, que aprobó el estudio, en 5 años revisó 356 protocolos, rechazó solo uno y en el año 2008 facturó por su "trabajo de revisión" 9,3 millones de dólares (78).

La bibliografía sobre los comités de ética en investigación es muy numerosa y se puede afirmar que, con excepción de unos pocos casos, la mayoría indica que estos tienen limitaciones para proteger a los sujetos y asegurar la calidad de los datos recogidos. En América Latina los estudios no son tantos pero suficientes para llegar a la misma conclusión.

En esta región es muy frecuente que los sujetos de investigación no entiendan los formularios de consentimiento informado y los CEI son indiferentes a esta realidad. En Costa Rica una evaluación de los comités locales de bioética realizada en 2009 descubrió que eran incapaces de desarrollar las funciones requeridas por el reglamento (79). En este mismo país, un ensayo clínico de la vacuna del cáncer de cérvix estuvo plagado de problemas éticos que los comités de ética fueron incapaces de evitar, por lo que fue objeto de denuncias en la Asamblea Legislativa $(80,81)$. La gravedad de la situación de los ensayos clínicos en Costa Rica condujo a que, en el año 
2010, la Corte Suprema prohibiera la aprobación de nuevos ensayos clínicos en humanos hasta que se aprobara una ley que regulara la experimentación clínica en humanos (79); legislación que se sancionó en abril de 2014. En Perú, un estudio demostró severas falencias en las evaluaciones realizadas por los CEI que podrían atribuirse tanto a falta de capacitación o negligencia, como a "deliberada y repetida" maniobra de omisión de información (82). En México, las severas falencias de los CEI fueron documentadas en una serie de ejemplares y detalladas investigaciones (83-86).

El Consejo Institucional de Revisión de Estudios de Investigación del Hospital Privado de Comunidad de Mar del Plata (Argentina) revisó, entre los años 2005 y 2006, 33 protocolos, hojas de información al paciente y consentimientos informados analizados previamente por "Comités de Ética Independientes" que persiguen fines de lucro (y que, por tanto, la demanda a ellos depende de la satisfacción de sus clientes), en los que se encontró tres objeciones relevantes por protocolo (76).

\section{CONCLUSIÓN Y PROPUESTAS}

Se ha explicado que la presencia de leyes y regulaciones no son suficientes para evitar violaciones de derechos humanos y de los principios éticos universalmente aceptados. Pero las violaciones empiezan en la elección misma de los temas a investigar, al abandonar el estudio de enfermedades que matan o dañan a millones de personas pero que no interesan al mercado del lucro, un aspecto de la investigación clínica que no está regulado.

Las agencias reguladoras son cómplices de que las regulaciones no se cumplan. Hay muchas causas por las que la legislación y las normas no se ejecutan, pero la influencia de la industria farmacéutica es la más importante. El resultado es que además de violarse los derechos humanos de los sujetos también se comercializan medicamentos que no son seguros ni eficaces, lo que constituye violaciones legales y éticas.

Se pueden hacer las siguientes propuestas para mejorar la situación en América Latina:

1) Es necesario concientizar a la ciudadanía de la importancia de la investigación médica y sobre sus derechos, a efectos de evitar su explotación. Sin participación ciudadana es poco probable que las normas se cumplan.

2) No debe aceptarse el doble estándar en investigación clínica.

3) La FDA y la EMA no son agencias confiables. Otros países no deben aprobar la comercialización de nuevos fármacos simplemente porque estos organismos lo hayan hecho. No es aceptable que las agencias reguladoras latinoamericanas se subordinen a las decisiones de la FDA y EMA.

4) Las agencias latinoamericanas reguladoras de medicamentos -la ANMAT, el Instituto Nacional de Vigilancia de Medicamentos y Alimentos (INVIMA), la Agência Nacional de Vigilância Sanitária (ANVISA), la Comisión Federal para la Protección contra Riesgos Sanitarios (COFREPIS), etc.- deben exigir, como condición necesaria para la aprobación de la comercialización de cualquier nueva entidad química, que los datos crudos de los estudios de investigación estén tanto a su disposición como de organismos e instituciones acreditados. No se debe invocar la excusa del secreto industrial para impedir una adecuada evaluación de la eficacia y seguridad de un nuevo fármaco.

5) Debería evaluarse, con participación de la ciudadanía y en forma transparente, la relación costo-beneficio antes de que un medicamento se incluya en los formularios de los servicios públicos y de los seguros sociales.

6) Se debe evitar que los funcionarios de las agencias reguladoras tengan conflictos de interés con la industria farmacéutica. Se requiere legislación que determine el número de años que debe pasar antes de que un funcionario pueda ser contratado por una industria farmacéutica, tras cesar en su puesto en la agencia, o de que un empleado de la industria sea contratado por la agencia.

7) Es necesario garantizar la transparencia en el funcionamiento de las agencias y elaborar mecanismos para que sean auditadas y sean sometidas al escrutinio público.

8) El funcionamiento de las agencias debe estar sometido a principios bioéticos claramente enmarcados en el respeto de los derechos humanos. 
9) Las agencias deberían Ilevar un registro de los estudios clínicos y epidemiológicos que se desarrollen en el territorio sobre el cual tengan jurisdicción. Los protocolos de investigación, las hojas de información y los consentimientos informados, con sus enmiendas, en sus distintas versiones e idiomas, deben ser archivados por las agencias con posibilidad de acceso público.

10) Los CEI deberían estar registrados en las agencias reguladoras. Se deben implementar los medios para asegurar que cumplan las funciones para las cuales han sido creados. No deberían acreditarse comités de ética comerciales ni los que se han creado como "fundaciones sin ánimo de lucro", cuya existencia depende de los ingresos que reciben por la aprobación de los documentos de los ensayos clínicos y que son, por tanto, dependientes de los patrocinadores.

11) Deben implementarse mecanismos claros de acreditación de los CEI así como instrumentos para asegurar las sanciones (tanto administrativas, económicas e incluso penales) para los que incumplen o transgreden la normativa existente.

12) Las decisiones de cada CEI deberían estar disponibles en las agencias. Cualquier rechazo, modificación o aceptación de protocolo, hoja de información o consentimiento informado tendría que ser automáticamente enviado al resto de los CEI y centros que realizan investigación.

13) La adulteración de datos, la manipulación de resultados, la promoción de falsa información de eficacia y seguridad de una nueva entidad química o medicamento tendrían que ser consideradas delitos contra los derechos humanos.

14) Es muy importante que cada país tenga una ley nacional de investigación biomédica que sancione (administrativa, económica e incluso penalmente) las violaciones de las normas que controlan la investigación clínica en humanos.

\section{REFERENCIAS BIBLIOGRÁFICAS}

1. Vollmann J, Winau R. Informed consent in human experimentation before the Nuremberg code. BMJ. 1996;313(7070):1445-1449.

2. Finlay CJ. El mosquito hipotéticamente considerado como agente de transmisión de la fiebre amarilla (1881). En: Buck C, Llopis A, Nájera E, Terris $M$, editores. El desafío de la epidemiología problemas y lecturas seleccionadas. Washington: Organización Panamericana de la Salud; 1994.

3. Lederer SE. Walter Reed and the Yellow Fever Experiments. En: Emanuel EJ, Crouch RA, Grady C, Lie RK, Miller FG, Wendler D, editores. The Oxford textbook of clinical research ethics. Oxford: Oxford University Press; 2008.

4. Sass HM. Reichsrundschreiben 1931: preNuremberg German regulations concerning new therapy and human experimentation. Journal of Medicine and Philosophy. 1983;8(2):99-111.

5. Lifton RJ. The Nazi doctors: medical killing and the psychology of genocide. New York: Basic Books; 1986.

6. Shevell M. Hallervorden and history. New England Journal of Medicine. 2003;348(1):3-4.
7. Tsuchiya T. The Imperial Japanese Experiments in China. En: Emanuel EJ, Crouch RA, Grady C, Lie RK, Miller FG, Wendler D, editors. The Oxford textbook of clinical research ethics. Oxford: Oxford University Press; 2008.

8. Tsuchiya T. Self determination by Imperial Japanese Doctors: Did they freely decide to perform deadly experiments? [Internet]. 2007 [citado 24 ago 2014]. Disponible en: http://www.lit. osaka-cu.ac.jp/user/tsuchiya/gyoseki/presentation/ UNESCOkumamoto07.html.

9. Homedes N, Ugalde A. Un estudio crítico de los principios éticos internacionales. En: Homedes $\mathrm{N}$, Ugalde A, coordinadores. Ética y ensayos clínicos en América Latina. Buenos Aires: Lugar Editorial; 2012.

10. Reverby SM. "Normal Exposure" and Inoculation Syphilis: A PHS "Tuskegee" Doctor in Guatemala, 1946-1948. Journal of Policy History. 2011;23(1):6-28

11. Jones $\mathrm{JH}$. The Tuskegee syphilis experiment. En: Emanuel EJ, Crouch RA, Grady C, Lie RK, Miller FG, Wendler D, editors. The Oxford textbook of clinical research ethics. Oxford: Oxford University Press; 2008. 
12. Reverby S. Examining Tuskegee: the infamous syphilis study and its legacy. Chapel Hill: University of North Carolina Press; 2009.

13. Reverby S. Tuskegee's truths: rethinking the Tuskegee syphilis study. Chapel Hill: University of North Carolina Press; 2000.

14. US scientists 'knew Guatemala syphilis tests unethical'. BBC News Latin America \& Caribbean [Internet]. 2011 [citado 20 set 2014]. Disponible en: http://www.bbc.com/news/world-latinamerica- 14712089.

15. Krugman S, Giles JP, Hammond J. Infectious hepatitis: Evidence for two distinctive clinical, epidemiological, and immunological types of infection. JAMA. 1967;200(5):365-373.

16. Beecher HK. Ethics and clinical research. New England Journal of Medicine. 1966;274(24): 1354-1360.

17. Goldby S. Experiments at the Willowbrook State School. Lancet. 1971;1(7702):749.

18. Krugman S. Experiments at the Willowbrook State School. Lancet. 1971;1(7706):966-967.

19. Robinson WM, Unruh BT. The Hepatitis experiments at the Willowbrook State School. En: Emanuel EJ, Crouch RA, Grady C, Lie RK, Miller FG, Wendler D, editors. The Oxford textbook of clinical research ethics. Oxford: Oxford University Press; 2008.

20. Arras JD. The Jewish Chronic Disease Hospital Case. En: Emanuel EJ, Crouch RA, Grady C, Lie RK, Miller FG, Wendler D, editors. The Oxford textbook of clinical research ethics. Oxford: Oxford University Press; 2008.

21. Khatchadourian R. Operación Delirium: Décadas después de experimentos peligrosos durante los años de la Guerra Fría, un científico vive con sus secretos. Boletín Fármacos[Internet]. 2013;16(1):64-71 [citado 15 oct 2014]. Disponible en: http://www.saludyfarmacos.org/wp-content/ files//feb2013.pdf.

22. Reuters. Nigeria insistirá con millonaria demanda contra Laboratorio Pfizer. Economía y Negocios online [Internet]. 2007 [citado 15 oct 2014]. Disponible en: http://www.economiaynegocios.cl/noticias/noticias.asp? $\mathrm{id}=30003$.

23. Umar A. Nigeria: Pfizer - Victims' Medical Records Missing. AllAfrica [Internet]. 2009 [citado 31 oct 2014]. Disponible en: http://allafrica.com/ stories/200910050887.html.
24. Goldacre B. Bad pharma: how drug companies mislead doctors and harm patients. London: Fourth Estate; 2012.

25. Lenzer J. Secret report surfaces showing that Pfizer was at fault in Nigerian drug tests. BMJ. 2006;332(7552):1233.

26. Lenzer J. Appeals court rules that Nigerian families can sue Pfizer in US. BMJ. 2009;338:b458.

27. Lee SB. Informed consent: Enforcing pharmaceutical companies' obligations abroad. Health and Human Rights. 2010;12(1):15-28.

28. Boseley S. WikiLeaks cables: Pfizer 'used dirty tricks to avoid clinical trial payout'. The Guardian [Internet]. 2010 [citado 10 oct 2014]. Disponible en: http://www.theguardian.com/business/2010/ dec/09/wikileaks-cables-pfizer-nigeria.

29. Homedes N, Ugalde A. Marco regulatorio y ensayos clínicos en Argentina. En: Homedes N, Ugalde A, coordinadores. Ética y ensayos clínicos en América Latina. Buenos Aires: Lugar Editorial; 2012.

30. CHE-CIREI. Rechazo del estudio "Bay 11643": Análisis crítico de las falencias metodológicas y éticas del protocolo. [Internet]. 2012 [citado 27 sep 2014]. Disponible en: http://www.hpc.org.ar/ images/adjuntos/842-BAY11643.pdf.

31. Ugalde A, Homedes N. Política y ensayos clínicos en la provincia de Córdoba. En: Homedes N, Ugalde A, coordinadores. Ética y ensayos clínicos en América Latina. Buenos Aires: Lugar Editorial; 2012.

32. Connor EM, Sperling RS, Gelber R, Kiselev P, Scott G, O'Sullivan MJ, et al. Reduction of maternal-infant transmission of human immunodeficiency virus type 1 with zidovudine treatment. New England Journal of Medicine. 1994;331(18):1173-1180.

33. Lurie $\mathrm{P}$, Wolfe $S M$. Unethical trials of interventions to reduce perinatal transmission of the human immunodeficiency virus in developing countries. New England Journal of Medicine. 1997;337(12):853-856.

34. Wiktor SZ, Ekpini E, Karon JM, Nkengasong J, Maurice C, Severin ST, Roels TH, Kouassi MK, Lackritz EM, Coulibaly IM, Greenberg AE. Short-course oral zidovudine for prevention of mother-to-child transmission of HIV-1 in Abidjan, Côte d'Ivoire: a randomised trial. Lancet. 1999;353(9155):781-785.

35. Shaffer N, Chuachoowong R, Mock PA, Bhadrakom C, Siriwasin W, Young NL, Chotpitaya- 
sunondh T, Chearskul S, Roongpisuthipong A, Chinayon P, Karon J, Mastro TD, Simonds RJ. Shortcourse zidovudine for perinatal HIV-1 transmission in Bangkok, Thailand: a randomised controlled trial, Bangkok Collaborative Perinatal HIV Transmission Study Group. Lancet. 1999;353(9155):773-780.

36. Consejo de Organizaciones Internacionales de las Ciencias Médicas. Pautas Éticas Internacionales para la Experimentación Biomédica en Seres Humanos. Ginebra: CIOMS, OMS; 1993.

37. Angell $M$. The ethics of clinical research in the Third World. New England Journal of Medicine. 1997;337(12):847-849.

38. Asociación Médica Mundial. Declaración de Helsinki: Guía de recomendaciones a los especialistas en investigaciones biomédicas que involucren a seres humanos. Hong Kong: Asamblea Médica Internacional; 1989.

39. European Medicines Agency. ICH Topic E 10, Choice of Control Group in Clinical Trials: note for guidance on choice of control group in clinical trials, CPMP/ICH/364/96 [Internet]. 2001 [citado 19 sep 2014]. Disponible en: http://www.ema. europa.eu/docs/en_GB/document_library/Scientific_guideline/2009̄/09/WC500002925.pdf.

40. Public Citizen. Request to the Department of Health and Human Services to halt plans for unethical placebo-controlled study of drug for respiratory distress syndrome in Latin America unless it is redesigned to treat all patients (HRG Publication \#1558) [Internet]. 2001 [citado 19 may 2013]. Disponible en: http://www.citizen.org/publications/ publicationredirect.cfm? ID $=6761$.

41. Soll RF. Synthetic surfactant for respiratory distress syndrome in preterm infants. Cochrane Database of Systematic Reviews. 2000;(2):CD001149.

42. Lurie P. Estudios sobre "Surfaxin" en America Latina. [Internet]. 2001 [citado 1 nov 2014]. Disponible en: http://www.essentialdrugs.org/efarmacos/archive/200104/msg00011.php.

43. Anderson M. FDA abandons Declaration of Helsinki for International Trials. The Social Medicine Portal [Internet]. 2008 [citado 19 may 2013]. Disponible en: http://www.socialmedicine. org/2008/06/01/ethics/fda-abandons-declarationof-helsinki-for-international-clinical-trials/.

44. Department of Health and Human Services, Food and Drug Administration. Federal Register: 21 CFR Part 312, Human Subject Protection; Foreign clinical studies not conducted under an investigational new drug Application. Rules and Regulations [Internet]. 2008 [citado 1 nov 2014];73(82):22800-
22816. Disponible en: http://www.fda.gov/ OHRMS/DOCKETS/98fr/E8-9200.htm.

45. Shah S. Cazadores de cuerpo. Madrid: 451 Editores; 2009.

46. Elliott C. White coat, black hat: adventures on the dark side of medicine. Boston: Beacon Press; 2010.

47. Cohen J, Dibner MS, Wilson A. Development of and access to products for neglected diseases. PloS One. 2010;5(5):e10610.

48. Trouiller P, Olliaro P, Torreele E, Orbinski J, Laing R, Ford N. Drug development for neglected diseases: a deficient market and a public-health policy failure. Lancet. 2002;359(9324):2188-2194.

49. BellS. From practiceresearch to publicpolicy: The ministerial summit on health research. The Annals of Pharmacotherapy. 2005;39(7):1331-1335.

50. Angell M. Excess in the pharmaceutical industry. CMAJ. 2004;171(12):1451-1453.

51. Donovan GK. Ebola, epidemics, and ethics: what we have learned. Philosophy, Ethics, and Humanities in Medicine. 2014;9(1):15.

52. Angell M. La verdad acerca de la industria farmacéutica: cómo nos engaña y qué hacer al respecto. Bogotá: Grupo Editorial Norma; 2006.

53. Gøtzsche PC. Corporate crime in the pharmaceutical industry is common, serious and repetitive [Internet]. 2012 [citado 31 oct 2014]. Disponible en: http://www.cochrane.dk/research/corporatecrime/Corporate-crime-long-version.pdf.

54. Krumholz HM. Neuraminidase inhibitors for influenza. BMJ. 2014;348:g2548.

55. Heneghan CJ, Onakpoya I, Thompson M, Spencer EA, Jones M, Jefferson T. Zanamivir for influenza in adults and children: systematic review of clinical study reports and summary of regulatory comments. BMJ. 2014;348:g2547.

56. Jefferson $T$, Jones $M$, Doshi $P$, Spencer $E A$, Onakpoya I, Heneghan CJ. Oseltamivir for influenza in adults and children: systematic review of clinical study reports and summary of regulatory comments. BMJ. 2014;348:g2545.

57. Jefferson $T$, Jones $M A$, Doshi $P$, Del Mar CB, Hama R, Thompson MJ, Onakpoya I, Heneghan CJ. Risk of bias in industry-funded oseltamivir trials: comparison of core reports versus full clinical study reports. BMJ open. 2014;4(9):e005253. 
58. Abbasi K. The missing data that cost $\$ 20 b n$. BMJ 2014;348:g2695.

59. Cañás $M$, Ugalde $A$, Orchuela J, Homedes N. Las secuelas del rofecoxib. Boletín Fármacos [Internet]. 2005;8(2):58-70 [citado 10 oct 2014]. Disponible en: http://www.saludyfarmacos.org/ wp-content/files//apr05.pdf.

60. Greener M. First do no harm: Improving drug safety through legislation and independent research. EMBO reports. 2008;9(3):221-224.

61. Graham DJ. Testimony of David J. Graham, MD, MPH, November 18, 2004 [Internet]. 2004 [citado 10 nov 2014]. Disponible en: http://www.finance. senate.gov/imo/media/doc/111804dgtest.pdf.

62. Kondro W, Sibbald B. Drug company experts advised staff to withhold data about SSRI use in children. CMAJ. 2004;170(5):783.

63. Healy D. Did regulators fail over selective serotonin reuptake inhibitors? BMJ. 2006;333(7558):92-95.

64. Healy D. Pharmageddon. Berkeley: University of California Press; 2012.

65. Angell M. Industry-sponsored clinical research: A broken system. JAMA. 2008;300(9):1069-1071.

66. Gonorazky SE. La injerencia de la industria farmacéutica en las actividades científicas. Neurología Argentina. 2013;5(3):141-148.

67. Psaty BM, Kronmal RA. Reporting mortality findings in trials of rofecoxib for Alzheimer disease or cognitive impairment: a case study based on documents from rofecoxib litigation. JAMA. 2008;299(15):1813-1817.

68. McGauran N, Wieseler B, Kreis J, Schuler YB, Kolsch $\mathrm{H}$, Kaiser T. Reporting bias in medical research: a narrative review. Trials. 2010;11:37.

69. Gøtzsche PC. We need access to all data from all clinical trials. Cochrane Database of Systematic Reviews. 2011;(12):ED000035.

70. Hrynaszkiewicz I, Altman DG. Towards agreement on best practice for publishing raw clinical trial data. Trials. 2009;10:17.

71. Hrynaszkiewicz I, Norton ML, Vickers AJ, Altman DG. Preparing raw clinical data for publication: guidance for journal editors, authors, and peer reviewers. Trials. 2010;11:9.

72. Wieseler B, McGauran N, Kerekes MF, Kaiser $\mathrm{T}$. Access to regulatory data from the European
Medicines Agency: the times they are a-changing. Systematic Reviews. 2012;1:50.

73. Cauchon D. FDA advisors tied to industry. USA TODAY [Internet]. 2000 [citado 10 oct 2014]. Disponible en: http://www.toolsforhealing.com/CD/ Articles/F/FDAAdvisorsTiedtolndustry.html.

74. Levinson DR. The Food And Drug Administration's oversight of clinical trials. [Internet]. 2007 [citado 20 ago 2010]. Disponible en: http://oig.hhs.gov/oei/reports/oei-01-06-00160. pdf.

75. Levinson DR. Challenges to FDA's ability to monitor and inspect foreign clinical trials [Internet]. 2010 [citado 20 ago 2014]. Disponible en: http:// oig.hhs.gov/oei/reports/oei-01-08-00510.pdf.

76. Gonorazky SE. Comités de ética independientes para la investigación clínica en la Argentina: Evaluación y sistema para garantizar su independencia. Medicina (Buenos Aires). 2008;68(2):113-119.

77. United States Government Accountability Office. Human Subjects Research: Undercover tests show the institutional review board system is vulnerable to unethical manipulation [Internet]. 2009 [citado 4 oct 2014]. Disponible en: http:// www.gao.gov/new.items/d09448t.pdf.

78. Meier B. An overseer of trials in medicine draws fire. The New York Times [Internet]. 2009 [citado 15 oct 2014]. Disponible en: http://www.nytimes. com/2009/03/27/business/27clinic.html? $r=0$.

79. Ugalde A, Homedes N. Un pequeño país para las grandes farmacéuticas: los ensayos clínicos en Costa Rica. En: Homedes N, Ugalde A, coordinadores. Ética y ensayos clínicos en América Latina. Buenos Aires: Lugar Editorial; 2012.

80. Costa Rica: Diputados denuncian impunidad en experimentación con seres humanos. Boletín Fármacos [Internet]. 2006;9(3):93-95 [citado 10 oct 2014]. Disponible en: http://www.saludyfarmacos.org/wp-content/files//jun06.pdf.

81. Homedes N, Ugalde A. El cáncer de cérvix y el desarrollo de vacunas para el control del VPH en Guanacaste, Costa Rica. En: Homedes N, Ugalde A, coordinadores. Ética y ensayos clínicos en América Latina. Buenos Aires: Lugar Editorial; 2012.

82. Minaya-Martínez G, Díaz-Sandoval R. Capacidad de evaluación de los ensayos clínicos por los Comités de Ética. Revista de la Sociedad Peruana de Medicina Interna. 2008;21(4):153-165. 
83. Valdez-Martinez E, Garduno-Espinosa J, Martinez-Salgado H, Porter JD. Local research ethics committees of the Mexican Institute of Social Security: results of a national survey. Public Health. 2004;118(5):329-336.

84. Valdez-Martinez E, Trumbull B, GardunoEspinosa J, Porter JD. Understanding the structure and practices of research ethics committees through research and audit: a study from Mexico. Health Policy. 2005;74(1):56-68.
85. Valdez-Martinez E, Turnbull B, GardunoEspinosa J, Porter JD. Descriptive ethics: a qualitative study of local research ethics committees in Mexico. Developing World Bioethics. 2006;6(2):95-105.

86. Verástegui-Avilés E, Valdez-Martínez E. Regulación y ética de los ensayos clínicos en México. En: Homedes N, Ugalde A, coordinadores. Ética y ensayos clínicos en América Latina. Buenos Aires: Lugar Editorial; 2012.

\section{FORMA DE CITAR}

Gonorazky SE. Los principios éticos universales y su aplicación a los ensayos clínicos de medicamentos. Salud Colectiva. 2015;11(1):49-65.

Recibido: 15 de noviembre de 2014

Versión final: 20 de diciembre de 2014

Aprobado: 17 de enero de 2015 